\title{
Phytotoxicity, cytotoxicity and chemical composition of Spondias mombin Linn. Stem bark
}

\author{
Kissinger Obaogie Orumwensodia ${ }^{1,2^{*}}$ D, Patrick Ojeifo Uadia ${ }^{1}$ and Muhammed lqbal Choudhary ${ }^{2}$
}

\begin{abstract}
Background: Spondias mombin Linn. is a tropical climate plant with wide applications in ethnomedicinal practice. This study evaluates the phytotoxicity, cytotoxicity and chemical composition of the plant's stem bark.

Methods: Dried stem bark sample of Spondias mombin Linn. was subjected to exhaustive extraction and partitioned into sub-fractions (hexane-ethylacetate, ethylacetate, ethylacetate-methanol and methanol) by graded polarity technique. The phytotoxicity and cytotoxicity indices of the crude hydro-ethanol extract and fractions were evaluated using Lemna minor and brine shrimp lethality assays, respectively, while chemical composition of the oily hexane:ethylacetate fraction was determined by gas chromatography-mass spectroscopy (GC-MS) technique.

Results: Phytotoxicity was dose-dependent which ranged from low (crude plant extract), moderate (hexaneethylacetate and methanol fractions), high (ethylaacetate-methanol fraction) to significant toxicity (ethylacetate fraction) at the highest dose. However, for brine shrimp lethality assay only hexane-ethylacetate $\left(\mathrm{LD}_{50}: 284.02 \mu \mathrm{g} /\right.$ $\mathrm{mL})$ and ethylacetate $\left(\mathrm{LD}_{50}: 210.24 \mu \mathrm{g} / \mathrm{mL}\right)$ fractions were cytotoxic at the highest dose. The GC-MS profile of the oily hexane:ethylacetate fraction identified sixty-eight compounds comprising hydrocarbons, fatty acids, alcohols, steroids, nitrogen and fluoride-containing compounds, terpenes and esters.
\end{abstract}

Conclusion: This study concludes that fractions of Spondias mombin Lin. could be potentially toxic. While its phytotoxic potential can be useful in the agrochemical industry for the production of natural herbicides, its cytotoxic property can be cautiously harnessed for ethnomedicinal purposes.

Keywords: Spondias mombin, Phytotoxicity, Cytotoxicity, Lemna aequinocitalis Welv, Brine shrimp, GC-MS

\section{Background}

Spondias mombin Linn. is a tree belonging to the family of Anacardiaceae and subfamily Spondiadoideae. It grows within the humid tropical climates, often in secondary vegetation derived from evergreen lowland forest or semi-deciduous forest areas of the continents of Africa (in countries like Nigeria, Congo, Central Africa Republic, etc), Asia (India) and South Americas (Brazil, Guatemala, Panama, Argentina, etc.) [1]. Its common

\footnotetext{
* Correspondence: kissinger.orumwensodia@uniben.edu

'Department of Biochemistry, Faculty of Life Sciences, University of Benin, Benin City, Nigeria

${ }^{2} \mathrm{H}$. E. J. Research Institute of Chemistry, International Center for Chemical and Biological Sciences (ICCBS), University of Karachi, Karachi 75270, Pakistan
}

names include hog plum, yellow mombin, mombin and yellow Spanish plum. In Nigeria it is locally referred to as Ogheghe, Okighan in Edo, Tsáádàr Másàr in Hausa, Ijikara, Ngulungwu, Isikala in Igbo, Iyeye, Ekikan, Olosan in Yoruba, and Nsukakara in Ibibio [2]. It has been greatly exploited around the world for various purposes including ornamental, nutritional (as a beverage) and medicinal; anti-malarial [3], antiviral [4], antibacterial [5], wound-healing [6], enzyme inhibition [7], etc. The fruit hosts considerable amounts of vitamins $\mathrm{A}$ and $\mathrm{C}$, while carotenoids are presumably present in reasonable concentrations [8]. Qualitative phytochemical screening of parts of the plant revealed the presence of flavonoids, alkaloids, tannins, phenolics, saponins and

\section{Springer Open}

() The Author(s). 2021 Open Access This article is licensed under a Creative Commons Attribution 4.0 International License, which permits use, sharing, adaptation, distribution and reproduction in any medium or format, as long as you give appropriate credit to the original author(s) and the source, provide a link to the Creative Commons licence, and indicate if changes were made. The images or other third party material in this article are included in the article's Creative Commons licence, unless indicated otherwise in a credit line to the material. If material is not included in the article's Creative Commons licence and your intended use is not permitted by statutory regulation or exceeds the permitted use, you will need to obtain permission directly from the copyright holder. To view a copy of this licence, visit http://creativecommons.org/licenses/by/4.0/. 
proanthocyanins, which have been implicated in the healing potentials associated with medicinal plants like Spondias mombin. The use of these medicinal plants continues to gain grounds especially in lowincome countries. A WHO report on traditional medicine strategy for 2014-2023, opined that a good number of the world's population depend on medicinal plants for therapeutic remedies [9]. However, the ethno-pharmacological usage of medicinal plants including Spondias mombin has been overshadowed by toxicity concerns bothering on their safety. Phytotoxicity and cytotoxicity assays are two ready-to-use, less expensive and easy to apply laboratory tests used to determine the toxicity profile of plant samples including extracts/fractions/isolated compounds [10, 11]. For instance, brine shrimp of the brine shrimp lethality assay (an example of a cytotoxicity assay) is believed to have positive correlation with human nasopharyngeal carcinoma (KB cell line) [10, 11], therefore a plant material which shows toxicity towards it could be potentially relevant in anticancer drug formulation. On the other hand, phytotoxicity assay can serve the purpose of screening for plant materials with potential herbicidal activity [12], since some of these products are eco-friendly but toxic to weeds. Therefore, owing to the medicinal values associated with S. mombin locally and its wide applications, this study was designed to investigating the toxicity index and chemical profile of this plant species of Nigerian origin.

\section{Materials and methods Chemicals}

All solvents (hexane, ethylacetate, methanol, and ethanol) were of analytical grade and products of SigmaAldrich, Germany. While Paraquat and Etoposide, the reference drugs, were products of ICN Biomedical Inc., California, USA.

\section{Plant materials}

Stem bark of Spondias mombin Linn. was harvested from its trees in the forest area of Southwest region of Nigeria within the month of November. The plant material was authenticated by Dr. H. A. Akinnibosun and Dr. J. Irabor of the Department of Plant Biology and Biotechnology, where voucher No. UBHa210 was assigned and herbarium samples deposited at the herbarium of Department of Plant Biology and Biotechnology, University of Benin. The plant part was washed with water to remove earthy materials, air dried and pulverized $(<1 \mathrm{~mm})$ to obtain the crude powdered sample.

\section{Extraction and fractionation}

Air-dried stem bark of Spondias mombin Linn. (750 g) was subjected to successive maceration $(4$ days $\times 3)$ using $70 \%$ ethanol/water $(2.5 \mathrm{~L})$ at room temperature. The concentrated hydro-ethanol extract (31.7 g) was fractionated in a stepwise gradient pattern of increasing solvent polarity of hexane (100\%), hexane-ethylacetate (50:50), ethylacetate (100\%), ethylacetate-methanol (50:50) and methanol $(100 \%)$ to obtain hexane, hexane-ethylacetate, ethylacetate, ethylacetate-methanol and methanol soluble fractions under reduced pressure (20-200 mbar) using a rotavapor at $45^{\circ} \mathrm{C}$.

\section{Phytotoxicity assay}

The assay was done according to the modified methods of McLaughlin et al. [11]. Briefly, the extract/fractions were incorporated into sterilized conical flasks at varying concentrations of 10,100 , and $1000 \mu \mathrm{g} / \mathrm{mL}$ in methanol, and allowed to evaporate overnight. Each flask was inoculated with $20 \mathrm{~mL}$ of sterilized E-medium and 10 plants of Lemna aequinocitalis Welv. containing a roselle of two to three fronds. The E-medium was prepared by mixing several components, viz.; boric acid $(0.00286 \mathrm{~g} /$ $\mathrm{L})$, copper sulphate $(0.00022 \mathrm{~g} / \mathrm{L})$, potassium dihydrogen phosphate $(0.68 \mathrm{~g} / \mathrm{L})$, calcium nitrare $(1.180 \mathrm{~g} / \mathrm{L})$, potassium nitrate $(1.515 \mathrm{~g} / \mathrm{L})$, magnesium sulphate $(0.492 \mathrm{~g} /$ $\mathrm{L})$, magenous chloride $(0.00362 \mathrm{~g} / \mathrm{L})$, ferric chloride $(0.00540 \mathrm{~g} / \mathrm{L})$, zinc sulphate $(0.00022 \mathrm{~g} / \mathrm{L})$, sodium molybdate and ethylene diamino tetracetic acid, in $1000 \mathrm{~mL}$ distilled water with the $\mathrm{pH}$ adjusted to between 5.5-6.0 by adding $\mathrm{KOH}$ pellets and autoclaved at $121{ }^{\circ} \mathrm{C}$ for 15 min. The negative control flasks were supplemented with methanol, while the reference inhibitor, paraquat, served as positive control. The experiment was done in triplicates and the flasks incubated at $30^{\circ} \mathrm{C}$ for 7 days in a Fisons Fi-Totran $600 \mathrm{H}$ growth cabinet with experimental conditions set at $56 \pm 10$ rh (relative humidity), $12 \mathrm{~h}$ day length and $9000 \mathrm{~lx}$ light intensity. The growth of $L$. aequinocitalis in the treatment flasks was determined by counting the number of fronds per dose, while growth inhibition in percentage with reference to the negative control was determined as follows:

$$
\text { Growth regulation }(\%)=\frac{\text { Number of fronds in negative control-Number of fronds in test flasks }}{\text { Number of fronds in negative control }} \times 100
$$

\section{Brine shrimp lethality assay}

Brine shrimp lethality assay was performed according to the modified methods of Carballo et al. [12]. Briefly, the eggs of brine shrimp (Artemia salina), stored at $4{ }^{\circ} \mathrm{C}$, were hatched and shrimp between 48 and $72 \mathrm{~h}$ after the initiation of hatching were used for the experiment. Test samples (extract/fractions of Spondias mombin Linn. stem bark) of concentrations 10, 100, and $1000 \mu \mathrm{g} / \mathrm{mL}$ 
dissolved in methanol were introduced into their respective vials and the solvent allowed to evaporate over night. Subsequently, ten larvae per vial (about 2 day old shrimp, nauplii) were placed into the vials with the aid of a Pasteur pipette and the vials filled with $5 \mathrm{~mL}$ sea water. The set up was incubated at $28-29^{\circ} \mathrm{C}$ for $24 \mathrm{~h}$ under illumination. Vials with solvent served as negative control, while the reference drug, Etoposide, was used as positive control. The experiment was performed in triplicate. Cytotoxicity of extract/fractions was evaluated by counting the numbers of live and dead larvae and $\mathrm{LD}_{50}$ value was determined according to the formula below. Data obtained were analyzed using Finney computer program and confidence level set at 95\% confidence intervals.

$$
\mathrm{LD}_{50}=\frac{\sqrt{\mathrm{D}_{0} \mathrm{X} \mathrm{D}_{100}}}{2}
$$

$\mathrm{D}_{0}=$ Highest dose that gave no mortality

$\mathrm{D}_{100}=$ Lowest dose that produced mortality

Gas chromatography-mass spectrometry (GC-MS) analysis The GC-MS analysis of the hexane:ethylacetate fraction (viscous oil) of Spondias mombin Linn. stem bark was performed in a GC-MS-TQQQ instrument equipped with Agilent USB39375HHP-5MS column and capillary dimensions $30 \mathrm{~m} \times 250 \mu \mathrm{m} \times 0.25 \mu \mathrm{m}$. Helium was used as the carrier gas at a flow rate of $1.2 \mathrm{~mL} / \mathrm{min}$ and pressure was maintained at $10.97 \mathrm{psi}$, while the injection volume was $1 \mu \mathrm{L}$. The oven equilibration was for $30 \mathrm{~min}$ and temperature was pre-set at $70^{\circ} \mathrm{C}$ for $5 \mathrm{~min}$, the $10{ }^{\circ} \mathrm{C} / \mathrm{min}$ to $180^{\circ} \mathrm{C}$ for $5 \mathrm{~min}, 10^{\circ} \mathrm{C} / \mathrm{min}$ to $280{ }^{\circ} \mathrm{C}$ for $10 \mathrm{~min}$, and $5{ }^{\circ} \mathrm{C} / \mathrm{min}$ to $290^{\circ} \mathrm{C}$ for $30 \mathrm{~min}$. While, the MS transfer line was sustained at a temperature of $325^{\circ} \mathrm{C}$, the total run time was $73 \mathrm{~min}$. The ionization mode used was electron ionization at $70 \mathrm{eV}$ with source temperature of $250{ }^{\circ} \mathrm{C}$. Total Ion Count (TIC) was used for compound identification at start mass of $20 \mathrm{amu}$ and end mass of $650 \mathrm{amu}$ for scan time of $200 \mathrm{~ms}$. With Match Factor (MF) of $\geq 700$ taken as satisfactory, the Spectra of the separated compounds were compared with the database of the National Institute of Standards and Technology (NIST) Reference Spectra Library using AMDIS V 2.69 (Automated mass spectral deconvolution and identification software). The relative percentage compositions of the identified compounds were estimated from the GC peak area.

\section{Statistical analysis}

Data were expressed as percentage growth inhibition of three replicates. The data were subjected to one-way analysis of variance (ANOVA), and differences between means were determined by Duncan's multiple range test using the Statistical Analysis System (SPSS Statistics 20.0) where applicable. Significance was set at $P$ values $\leq 0.05$.

\section{Results}

\section{Phytotoxicity assay}

At a dose of $10 \mu \mathrm{g} / \mathrm{mL}$, all fractions and extract of Spondias mombin stem bark had zero inhibition growth effect on fronds of Lemna minor plant, while the methanol fraction had similar effect up to $100 \mu \mathrm{g} / \mathrm{mL}$. Conversely, aside paraquat (the reference drug) only ethylacetate fraction at the highest dose of $1000 \mu \mathrm{g} / \mathrm{mL}$ had a $100 \%$ growth inhibition. However, other fractions displayed varying degrees of growth inhibition. Results are presented in Table 1.

\section{Brine shrimp (Artemia salina) lethality assay}

Only Hexane:ethylacetate and ethylacetate fracetions had cytotoxic effect at the highest dose of $1000 \mu \mathrm{g} / \mathrm{mL}$. Other fractions including the crude hydro-ethanol extract demonstrated no cytotoxic effect. Results are presented in Table 2.

\section{Gas chromatography-mass spectrometry (GC-MS)}

The GC-MS chromatograms in Fig. 1a, b and c, revealed sixty-eight (68) peaks matching phytoconstituents in the class of hydrocarbons, fatty acids, alcohols, steroids, nitrogen and fluoride-containing compounds, terpenes and esters. Their molecular formula, molecular weight, retention time, peak area, and reverse match factor are presented in Table 3.

\section{Discussion}

The use of herbal preparations as potent therapeutic interventions predates modern medicine. Plants have been found to contain several bioactive principles with significant value in the drug formulation process. These bioactive principles otherwise referred to as phytochemicals

Table 1 Phytotoxic effect of Spondias mombin stem bark and Paraquat at various concentrations against fronds of Lemna minor

\begin{tabular}{llll}
\hline Test Samples & \multicolumn{3}{l}{ \% Growth regulation at different doses } \\
\cline { 2 - 4 } & $\mathbf{1 0} \boldsymbol{\mu \mathbf { g } / \mathbf { m L }}$ & $\mathbf{1 0 0} \boldsymbol{\mu \mathbf { g } / \mathbf { m L }}$ & $\mathbf{1 0 0 0} \boldsymbol{\mu \mathbf { g }} \mathbf{\mathbf { m L }}$ \\
\hline Hex:EA & 0.0 & $28.5 \pm 0.41$ & $59.5 \pm 0.33$ \\
EA & 0.0 & $14.0 \pm 0.20^{*}$ & 100.0 \\
EA:Met & 0.0 & $37.5 \pm 0.11$ & $65.6 \pm 0.20$ \\
Met & 0.0 & 0.0 & $52.4 \pm 0.10$ \\
CpE & 0.0 & $30.9 \pm 0.10$ & $38.1 \pm 0.25$ \\
Paraquat $(0.015 \mu \mathrm{g} / \mathrm{mL})$ & 100.0 & 100.0 & 100.0 \\
\hline
\end{tabular}

Values are mean \pm S.E.M $(n=3),{ }^{*} p<0.05$. Hex:EA Hexane:ethylacetate, EA Ethylacetate, EA:Met Ethylacetate:methanol, Met Methanol and $C p E$ Crude plant extract. Paraquat: reference drug 
Table 2 Cytotoxic effect of Spondias mombin stem bark and Etoposide at various concentrations against shrimps of Artemia salina

\begin{tabular}{|c|c|c|c|c|}
\hline \multirow{2}{*}{$\begin{array}{l}\text { Test } \\
\text { Samples }\end{array}$} & \multicolumn{3}{|c|}{ No. of survivals out of 30 shrimps at different doses } & \multirow{2}{*}{$\begin{array}{l}\mathrm{LD}_{50}(\mu \mathrm{g} / \\
\mathrm{mL})\end{array}$} \\
\hline & $10(\mu \mathrm{g} / \mathrm{mL})$ & $100(\mu \mathrm{g} / \mathrm{mL})$ & $1000(\mu \mathrm{g} / \mathrm{mL})$ & \\
\hline Hex:EA & 29 & 22 & 07 & $284.0 \pm 0.20$ \\
\hline EA & 28 & 27 & 01 & $210.2 \pm 0.15$ \\
\hline EA:Met & 28 & 26 & 24 & - \\
\hline Met & 29 & 28 & 27 & - \\
\hline $\mathrm{CpE}$ & 30 & 30 & 22 & - \\
\hline Etoposide & 00 & 00 & 00 & 7.5 \\
\hline
\end{tabular}

Values are mean \pm S.E.M $(n=3),{ }^{*} p<0.05$. Hex:EA Hexane:ethylacetate, EA Ethylacetate, EA:Met Ethylacetate:methanol, Met Methanol and CpE Crude plant extract. Etoposide: reference drug
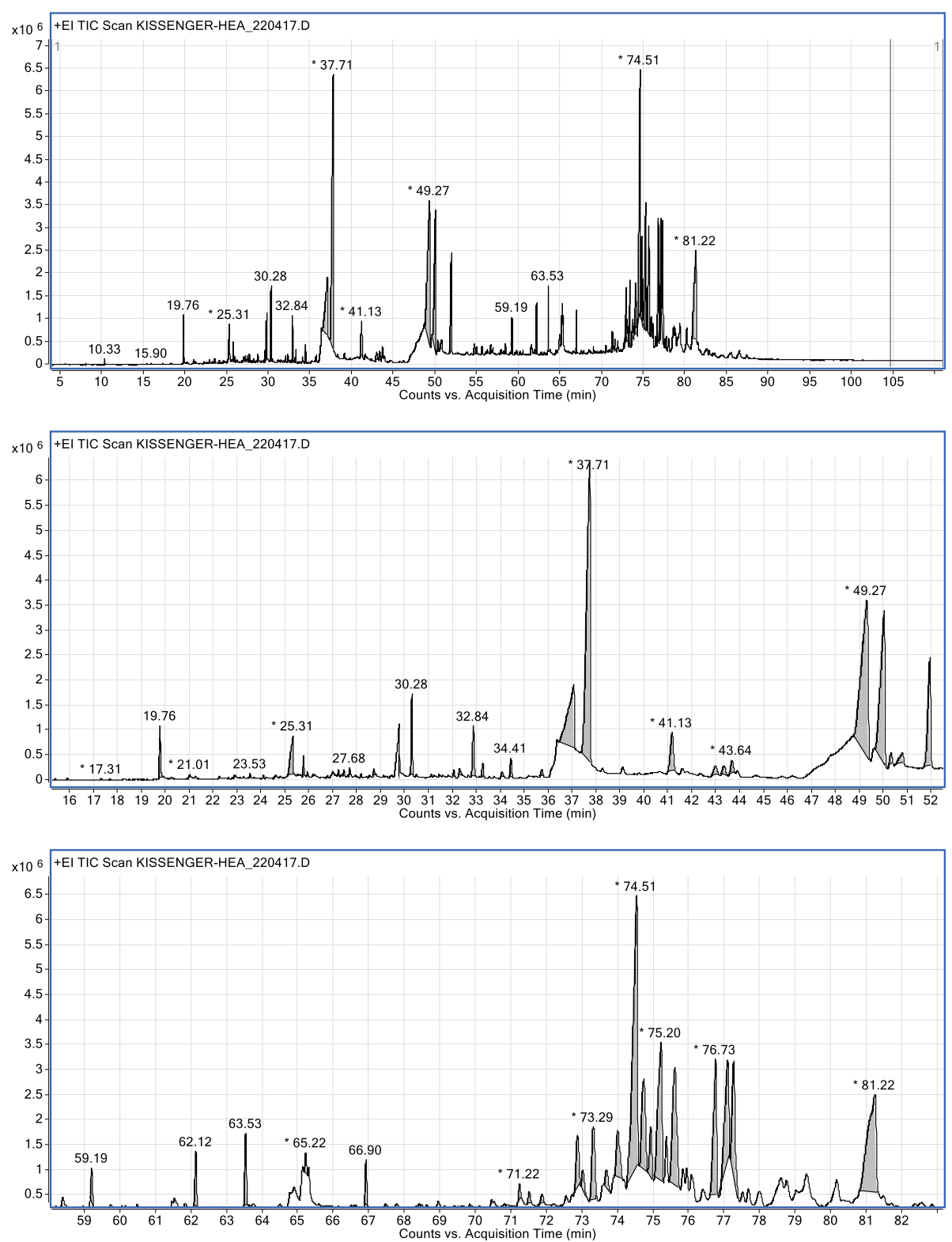

Fig. 1 Chromatogram of Phytoconstituents in Spondias mombin Linn. stem bark oil 
Table 3 Compounds identified in Spondias mombin stem bark oil

\begin{tabular}{|c|c|c|c|c|c|}
\hline Compound name & $\begin{array}{l}\text { Molecular } \\
\text { formula }\end{array}$ & MW & $\begin{array}{l}\text { RT } \\
(\min )\end{array}$ & $\begin{array}{l}\text { Peak } \\
\text { Area \% }\end{array}$ & $\begin{array}{l}\text { RMF } \\
\text { (DB) }\end{array}$ \\
\hline 2,3-Dimethyl-1-pentanol & $\mathrm{C}_{7} \mathrm{H}_{16} \mathrm{O}$ & 116 & 8.10 & 0.01 & 849 \\
\hline 2-Ethylhexan-1-ol & $\mathrm{C}_{8} \mathrm{H}_{18} \mathrm{O}$ & 130 & 10.33 & 0.06 & 943 \\
\hline 2-Propyl-1-heptanol & $\mathrm{C}_{10} \mathrm{H}_{22} \mathrm{O}$ & 158 & 15.90 & 0.01 & 835 \\
\hline (2E)-2-Tridecenal & $\mathrm{C}_{13} \mathrm{H}_{24} \mathrm{O}$ & 196 & 17.31 & 0.02 & 777 \\
\hline Eugenol & $\mathrm{C}_{10} \mathrm{H}_{12} \mathrm{O}_{2}$ & 164 & 19.76 & 0.01 & 943 \\
\hline d-Mannose & $\mathrm{C}_{6} \mathrm{H}_{12} \mathrm{O}_{6}$ & 180 & 20.25 & 0.72 & 741 \\
\hline Vanillin lactoside & $\mathrm{C}_{20} \mathrm{H}_{28} \mathrm{O}_{13}$ & 476 & 21.01 & 0.02 & 778 \\
\hline (Z)-7-Hexadecenal & $\mathrm{C}_{16} \mathrm{H}_{30} \mathrm{O}$ & 238 & 21.26 & 0.06 & 870 \\
\hline 6-Pentyl-5,6-dihydro-2H-pyran-2-one (Massoia lactone) & $\mathrm{C}_{10} \mathrm{H}_{16} \mathrm{O}_{2}$ & 168 & 22.88 & 0.02 & 842 \\
\hline Tetradecane, 2,6,10-trimethyl- & $\mathrm{C}_{17} \mathrm{H}_{36}$ & 240 & 23.53 & 0.05 & 827 \\
\hline Undecanoic acid, 10-methyl-, methyl ester & $\mathrm{C}_{13} \mathrm{H}_{26} \mathrm{O}_{2}$ & 214 & 24.08 & 0.03 & 866 \\
\hline Dodecanoic acid (Lauris Acid) & $\vdots \mathrm{C}_{12} \mathrm{H}_{24} \mathrm{O}_{2}$ & 200 & 25.31 & 0.02 & 908 \\
\hline Dodecanoic acid, ethyl ester (Ethyl laurate) & $\mathrm{C}_{14} \mathrm{H}_{28} \mathrm{O}_{2}$ & 228 & 25.75 & 0.04 & 932 \\
\hline Nonadecane & $\mathrm{C}_{19} \mathrm{H}_{40}$ & 268 & 25.99 & 1.24 & 927 \\
\hline 3,4,5-Trimethoxyphenol & $\mathrm{C}_{9} \mathrm{H}_{12} \mathrm{O}_{4}$ & 184 & 26.17 & 0.21 & 827 \\
\hline Octatriacontyl pentafluoropropionate & $\mathrm{C}_{41} \mathrm{H}_{77} \mathrm{~F}_{5} \mathrm{O}_{2}$ & 696 & 26.43 & 0.06 & 799 \\
\hline 2,2',5,5'-Tetramethyl-1,1'-biphenyl & $\mathrm{C}_{16} \mathrm{H}_{18}$ & 210 & 27.17 & 0.06 & 847 \\
\hline 1,4-Methanoazulen-3-ol, decahydro-1,5,5,8a-tetramethyl-, [1S-(1a,3ß,3aß,4a,8aß)]- (Longiborneol) & $\mathrm{C}_{15} \mathrm{H}_{26} \mathrm{O}$ & 222 & 26.76 & 0.02 & 746 \\
\hline 2-(2-Nitro-2-propenyl) cyclohexanone & $\mathrm{C}_{9} \mathrm{H}_{13} \mathrm{NO}_{3}$ & 183 & 26.98 & 0.05 & 746 \\
\hline Epiglobulol & $\mathrm{C}_{15} \mathrm{H}_{26} \mathrm{O}$ & 222 & 27.21 & 0.09 & 789 \\
\hline Globulol & $\mathrm{C}_{15} \mathrm{H}_{26} \mathrm{O}$ & 222 & 27.44 & 0.02 & 845 \\
\hline 1-Hexadecanol (Cetyl Alcohol) & $\mathrm{C}_{16} \mathrm{H}_{34} \mathrm{O}$ & 242 & 27.68 & 0.06 & 900 \\
\hline Tetradecyl trifluoroacetate & $\mathrm{C}_{16} \mathrm{H}_{29} \mathrm{~F}_{3} \mathrm{O}_{2}$ & 310 & 27.68 & 0.07 & 887 \\
\hline 2-Methyl-1-hexadecanol & $\mathrm{C}_{17} \mathrm{H}_{36} \mathrm{O}$ & 256 & 28.17 & 0.03 & 770 \\
\hline 3-Hydroxydodecanoic acid & $\mathrm{C}_{12} \mathrm{H}_{24} \mathrm{O}_{3}$ & 216 & 28.51 & 0.03 & 755 \\
\hline Tetradecanoic acid (Myristic acid) & $\mathrm{C}_{14} \mathrm{H}_{28} \mathrm{O}_{2}$ & 228 & 29.76 & 0.14 & 901 \\
\hline Tetradecanoic acid, ethyl ester (Myristic acid, ethyl ester) & $\mathrm{C}_{16} \mathrm{H}_{32} \mathrm{O}_{2}$ & 256 & 30.28 & 0.02 & 911 \\
\hline Hexadecanoic acid, ethyl ester (Palmitic acid, ethyl ester) & $\mathrm{C}_{18} \mathrm{H}_{36} \mathrm{O}_{2}$ & 284 & 32.02 & 0.98 & 785 \\
\hline Ethyl 13-methyl-tetradecanoate & $\mathrm{C}_{17} \mathrm{H}_{34} \mathrm{O}_{2}$ & 270 & 32.02 & 0.09 & 845 \\
\hline Oleic Acid (9-Octadecenoic acid (Z)-) & $\mathrm{C}_{18} \mathrm{H}_{34} \mathrm{O}_{2}$ & 282 & 32.27 & 0.18 & 763 \\
\hline 1-Hexadecanol & $\mathrm{C}_{16} \mathrm{H}_{34} \mathrm{O}$ & 242 & 32.84 & 0.96 & 946 \\
\hline Pentadecanoic acid, ethyl ester & $\mathrm{C}_{17} \mathrm{H}_{34} \mathrm{O}_{2}$ & 270 & 33.24 & 0.23 & 918 \\
\hline Ethyl (2E)-3-(4-hydroxy-3-methoxyphenyl)-2-propenoate (Ethyl ferulate) & $\mathrm{C}_{12} \mathrm{H}_{14} \mathrm{O}_{4}$ & 222 & 34.05 & 0.14 & 884 \\
\hline Docosanoic acid, ethyl ester & $\mathrm{C}_{24} \mathrm{H}_{48} \mathrm{O}_{2}$ & 368 & 35.71 & 0.37 & 754 \\
\hline n-Hexadecanoic acid & $\mathrm{C}_{16} \mathrm{H}_{32} \mathrm{O}_{2}$ & 256 & 37.03 & 0.16 & 929 \\
\hline Undecanoic acid, ethyl ester & $\mathrm{C}_{13} \mathrm{H}_{26} \mathrm{O}_{2}$ & 214 & 37.71 & 4.85 & 837 \\
\hline Oleyl Alcohol & $\mathrm{C}_{18} \mathrm{H}_{36} \mathrm{O}$ & 268 & 41.13 & 13.3 & 900 \\
\hline 11-Hexadecen-1-ol, (Z)- (Virelure) & $\mathrm{C}_{16} \mathrm{H}_{32} \mathrm{O}$ & 240 & 41.13 & 1.49 & 943 \\
\hline 1-Eicosanol & $\mathrm{C}_{20} \mathrm{H}_{42} \mathrm{O}$ & 298 & 42.95 & 0.42 & 908 \\
\hline Isopropyl Palmitate & $\mathrm{C}_{19} \mathrm{H}_{38} \mathrm{O}_{2}$ & 298 & 43.30 & 0.34 & 793 \\
\hline Heptadecanoic acid, ethyl ester & $\mathrm{C}_{19} \mathrm{H}_{38} \mathrm{O}_{2}$ & 298 & 43.64 & 0.49 & 826 \\
\hline 9,12-Octadecadienoic acid, ethyl ester & $\mathrm{C}_{20} \mathrm{H}_{36} \mathrm{O}_{2}$ & 308 & 49.27 & 12.47 & 910 \\
\hline 9-Octadecenoic acid, ethyl ester, (E)- & $\mathrm{C}_{20} \mathrm{H}_{38} \mathrm{O}_{2}$ & 310 & 50.28 & 7.87 & 864 \\
\hline
\end{tabular}


Table 3 Compounds identified in Spondias mombin stem bark oil (Continued)

\begin{tabular}{|c|c|c|c|c|c|}
\hline Compound name & $\begin{array}{l}\text { Molecular } \\
\text { formula }\end{array}$ & MW & $\begin{array}{l}\text { RT } \\
(\min )\end{array}$ & $\begin{array}{l}\text { Peak } \\
\text { Area \% }\end{array}$ & $\begin{array}{l}\text { RMF } \\
\text { (DB) }\end{array}$ \\
\hline Octadecanoic acid (Stearic acid) & $\mathrm{C}_{18} \mathrm{H}_{36} \mathrm{O}_{2}$ & 284 & 50.76 & 0.37 & 891 \\
\hline Methyl 17-methyl-octadecanoate & $\mathrm{C}_{20} \mathrm{H}_{40} \mathrm{O}_{2}$ & 312 & 51.90 & 0.59 & 869 \\
\hline Methyl 19-methyl-eicosanoate & $\mathrm{C}_{22} \mathrm{H}_{44} \mathrm{O}_{2}$ & 340 & 59.19 & 3.78 & 871 \\
\hline Eicosanoic acid, ethyl ester & $\mathrm{C}_{22} \mathrm{H}_{44} \mathrm{O}_{2}$ & 340 & 59.19 & 0.43 & 896 \\
\hline 1,2-Benzenedicarboxylic acid, diisooctyl ester (Isooctyl phthalate) & $\mathrm{C}_{24} \mathrm{H}_{38} \mathrm{O}_{4}$ & 390 & 62.12 & 0.68 & 951 \\
\hline 9-Octadecenoic acid (Z)-, 2-hydroxy-1-(hydroxymethyl)ethyl ester (Glyceryl 2-oleate) & $\mathrm{C}_{21} \mathrm{H}_{40} \mathrm{O}_{4}$ & 356 & 65.22 & 0.95 & 872 \\
\hline Ethyl tetracosanoate & $\mathrm{C}_{26} \mathrm{H}_{52} \mathrm{O}_{2}$ & 396 & 66.90 & 0.25 & 814 \\
\hline $\begin{array}{l}\text { 17-(1,5-Dimethylhexyl)-10,13-dimethyl-2,3,4,7,8,9,10,11,12,13,14,15,16,17-tetradecahydro-1H- } \\
\text { cyclopenta[a]phenanthren-3-ol }\end{array}$ & $\mathrm{C}_{27} \mathrm{H}_{46} \mathrm{O}$ & 386 & 71.22 & 0.5 & 867 \\
\hline Vitamin $\mathrm{E}$ & $\mathrm{C}_{29} \mathrm{H}_{50} \mathrm{O}_{2}$ & 430 & 71.49 & 0.28 & 827 \\
\hline Ethyl iso-allocholate & $\mathrm{C}_{26} \mathrm{H}_{44} \mathrm{O}_{5}$ & 436 & 71.86 & 0.23 & 801 \\
\hline Rhodopin & $\mathrm{C}_{40} \mathrm{H}_{58} \mathrm{O}$ & 554 & 71.86 & 0.23 & 733 \\
\hline Campesterol & $\mathrm{C}_{28} \mathrm{H}_{48} \mathrm{O}$ & 400 & 72.86 & 1.28 & 841 \\
\hline Stigmasterol & $\mathrm{C}_{29} \mathrm{H}_{48} \mathrm{O}$ & 412 & 73.29 & 0.35 & 918 \\
\hline Ergosta-5,24(28)-dien-3ß-ol (Chalinasterol) & $\mathrm{C}_{28} \mathrm{H}_{46} \mathrm{O}$ & 398 & 73.66 & 1.94 & 845 \\
\hline 4,14-Dimethylergosta-8,24(28)-dien-3-ol (Obtusifoliol) & $\mathrm{C}_{30} \mathrm{H}_{50} \mathrm{O}$ & 426 & 73.99 & 0.4 & 882 \\
\hline Y-Sitosterol & $\mathrm{C}_{29} \mathrm{H}_{50} \mathrm{O}$ & 414 & 74.51 & 1.39 & 927 \\
\hline$\beta$-Sitosterol & $\mathrm{C}_{29} \mathrm{H}_{50} \mathrm{O}$ & 414 & 74.51 & 10.01 & 916 \\
\hline $\begin{array}{l}\text { Cholest-5-en-3-ol, 24-propylidene-, (3ß)- ((E)-24-Propylidenecholesterol or 29- } \\
\text { Methylisofucosterol) }\end{array}$ & $\mathrm{C}_{30} \mathrm{H}_{50} \mathrm{O}$ & 426 & 74.72 & 2.76 & 812 \\
\hline Betulin & $\mathrm{C}_{30} \mathrm{H}_{50} \mathrm{O}_{2}$ & 442 & 74.72 & 0.92 & 747 \\
\hline Ergosta-7,24(28)-dien-3-ol, 4-methyl-, (3ß,4a,5a)- (Gramisterol) & $\mathrm{C}_{29} \mathrm{H}_{48} \mathrm{O}$ & 412 & 74.91 & 4.5 & 860 \\
\hline $\begin{array}{l}\text { 9,19-Cycloergost-24(28)-en-3-ol, 4,14-dimethyl-, acetate (9,19-Cycloergost-24(28)-en-3-ol, 4,14- } \\
\text { dimethyl-, acetate, }(3 \beta, 4 a, 5 a) \text {-) }\end{array}$ & $\mathrm{C}_{32} \mathrm{H}_{52} \mathrm{O}_{2}$ & 468 & 75.59 & 4.15 & 815 \\
\hline 24-Methylenecycloartan-3-one & $\mathrm{C}_{31} \mathrm{H}_{50} \mathrm{O}$ & 438 & 76.73 & 3.47 & 858 \\
\hline Stigmast-4-en-3-one (Sitostenone) & $\mathrm{C}_{29} \mathrm{H}_{48} \mathrm{O}$ & 412 & 77.08 & 3.61 & 908 \\
\hline 19-Cyclolanostan-3-ol, 24-methylene-, (3ß)- & $\mathrm{C}_{31} \mathrm{H}_{52} \mathrm{O}$ & 440 & 77.24 & 2.57 & 919 \\
\hline Stigmastane-3,6-dione, (5a)- & $\mathrm{C}_{29} \mathrm{H}_{48} \mathrm{O}_{2}$ & 428 & 81.22 & 6.88 & 866 \\
\hline
\end{tabular}

GC-MS was done using 'Agilent GC-MS triple quad USB39375HHP-5MS. The identification of compounds was based on a mass spectral survey performed using NIST library for spectral comparison and identification

are classed into saponins, tannins, flavonoids, phenolics, glycosides, organic acids, essential oils etc., and are believed to play a key role in the plant defense mechanism against invading pathogens. More so, several biological activities including antioxidant, anti-inflammatory, antibacterial, antifungal, enzyme modulation, as well as inhibition of cell proliferation amongst others have also been associated with these phytoconstituents [13]. Functioning as a sole molecule or in synergistic fashion, these potential drug candidates have helped to arrest several ailments [14-16]. Despite these seeming advantages, consumption of herbal formulations has been dabbed in controversies around safety issues. Therefore, scientific approaches that test the safety or otherwise of these products are required to resolve this conundrum. The result of phytotoxicity study of stem bark of Spondias mombin against L. aequinoctialis Welv. (Lemna minor)
(Table 1) indicates a possible phytotoxic effect at the highest tested dose of $1000 \mu \mathrm{g} / \mathrm{mL}$, relative to the reference drug, Paraquat. The ethylacetate fraction was significantly phytotoxic against fronds of Lemna minor plant at the highest dose tested. This was followed by ethylacetate:methanol fraction with high phytotoxic activity. Hexane:ethylacetate and methanol fractions both had moderate activity, while the crude hydro-ethanol extract showed weak phytotoxicity. Plants with phytotoxic activity have been exploited for use as natural herbicides [17]. Thus, the phytotoxic potential of Spondias mombin stem bark can be harnessed by agrochemical industries for the formulation of natural herbicides. Similarly, the result of brine shrimps lethality test (Table 2) shows some fractions had cytotoxic effect against Artemia salina at the highest dose of $1000 \mu \mathrm{g} / \mathrm{mL}$. Although, the crude hydro-ethanol extract, ethylacetate:methanol and 
methanol fractions demonstrated no cytotoxic activity relative to the reference drug, Etoposide, the hexane: ethylacetate and ethylacetate fractions had cytotoxic effect against Artemia salina. These findings, though on the stem bark of the plant, are in agreement with in vivo studies conducted on the aqueous and ethanolic leaf extracts of $S$. mombin, which revealed that prolonged usage of this plant at high doses could be potentially cytotoxic $[18,19]$. The cytotoxic property of some fractions of Spondias mombin stem bark at high concentration underscores the need for cautious use of the plant in ethno-medicinal practice. Nonetheless, phytoconstituents contained in the plant as revealed in this study via the GC-MS profiling of the oily hexane:ethylacetate fraction (Table 3 and Figs. 1, 2a, b, c) indicates a rich array of compounds, some of which have diverse pharmacological potentials. Sixty-eight compounds comprising hydrocarbons, fatty acids, alcohols, steroids, nitrogen and fluoride-containing compounds, terpenes and esters were identified (Figs. 1, 2a, b, c). These compounds include 2, 3-Dimethyl-1-pentanol (1); 2-Ethylhexan-1-ol (2); 2-Propyl-1-heptanol (3); (2E)-2-Tridecenal (4); Eugenol (5); d-Mannose (6); Vanillin lactoside (7), (Z)-7Hexadecenal (8); Massoia lactone (9); Tetradecane, 2,6, 10-trimethyl- (10); Undecanoic acid, 10-methyl-, methyl ester (11); Dodecanoic acid (Lauris Acid) (12); Dodecanoic acid, ethyl ester (Ethyl laurate) (13); Nonadecane (14); 3,4,5-Trimethoxyphenol (15); Octatriacontyl pentafluoropropionate (16); 2,2',5,5' -Tetramethyl-1,1'-biphenyl (17); Longiborneol (18); 2-(2-Nitro-2-propenyl) cyclohexanone (19); Epiglobulol (20); Globulol (21); Cetyl Alcohol (22); Tetradecyl trifluoroacetate (23); 2Methyl-1-hexadecanol (24); 3-Hydroxydodecanoic acid (25); Myristic acid (26); Myristic acid, ethyl ester (27); Palmitic acid, ethyl ester (28); Ethyl 13-methyltetradecanoate (29); Oleic Acid (30); 1-Hexadecanol (31); Pentadecanoic acid, ethyl ester (32); Ethyl ferulate (33); Docosanoic acid, ethyl ester (34); n-Hexadecanoic acid (35); Undecanoic acid, ethyl ester (36); Oleyl Alcohol (37); Virelure (38); 1-Eicosanol (39); Isopropyl Palmitate (40); Heptadecanoic acid, ethyl ester (41); 9, 12-Octadecadienoic acid, ethyl ester (42); 9Octadecenoic acid, ethyl ester, (E)- (43); Stearic acid (44); Methyl 17-methyl-octadecanoate (45); Methyl 19methyl-eicosanoate (46); Eicosanoic acid, ethyl ester (47); Isooctyl phthalate (48); Glyceryl 2-oleate (49); Ethyl tetracosanoate $\quad \mathbf{5 0}) ; \quad$ 17-(1,5-Dimethylhexyl)-10,13-dimethyl-2,3,4,7,8,9,10,11,12,13,14,15,16,17-tetradecahydro-1H-cyclopenta[a]phenanthren-3-ol (51); Vitamin E (52); Ethyl iso-allocholate (53); Rhodopin (54); Campesterol (55); Stigmasterol (56); Chalinasterol (57); Obtusifoliol (58); $\gamma$-Sitosterol (59); $\beta$-Sitosterol (60); Cholest-5en-3-ol, 24-propylidene-, (3ß)- (61); Betulin (62); Gramisterol (63); 9,19-Cycloergost-24(28)-en-3-ol, 4,14-
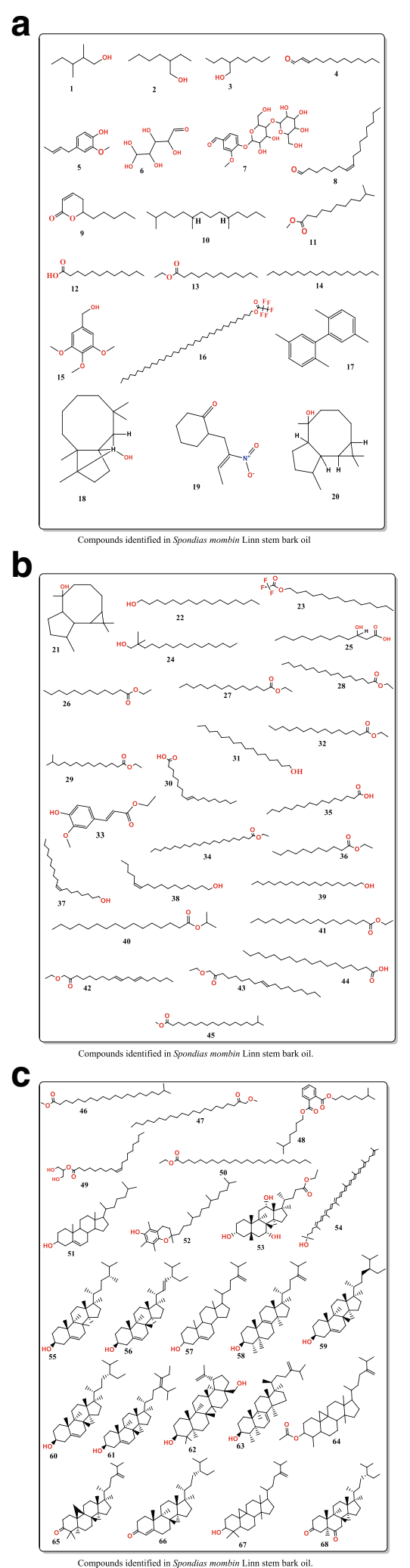

Fig. 2 a Compounds identified in Spondias mombin Linn stem bark oil. b Compounds identified in Spondias mombin Linn stem bark oil. c Compounds identified in Spondias mombin Linn stem bark oil 
dimethyl-, acetate (9,19-Cycloergost-24(28)-en-3-ol, 4, 14-dimethyl-, acetate, $(3 \beta, 4 \alpha, 5 \alpha)-) \quad(64) ; \quad 24-$ Methylenecycloartan-3-one (65); Sitostenone (66); 19-

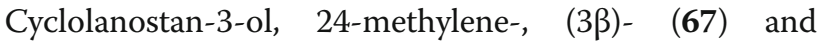
Stigmastane-3,6-dione, (5 $\alpha)$ - (68) (Fig. 2a, b, c). Some of these compounds as earlier mentioned have been found to possess profound biological activities. For instance, the long chain fatty acid alcohol, (2E)-2-Tridecenal, is known for its antibacterial activity [20]. Eugenol, which belongs to the class of allylbenzene and a naturally occurring phenolic molecule has anti-inflammatory, neuroprotective, antipyretic, antioxidant, antifungal and analgesic properties [21-23], antiproliferative and proapoptotic activity [24] and antimicrobial property [25]. Aside its pharmacological importance [26], reported the herbicidal role of eugenol in commercially available herbicide, clove oil (a herbicide formulation of Burnout II weed and grass killer). Therefore, its phytotoxic effect could be due to the presence of compounds like eugenol. Fatty acids such as oleic acid enhances membrane function [27], while stearic acid regulates mitofusin activity, ditto mitochondrial morphology and function, reduces blood pressure, improves heart function, and reduces cancer risk [28]. Some phytosterols such as campesterol, gramisterol and stigmasterol were found to promote WEHI-3 cell anti-proliferative activity, antiinflammatory effect and cytotoxicity against some cancer cell lines [29]. Thus, the cytotoxic effect of this plant could be linked to in part, its fatty acid and phytosterol contents amongst other molecules. Several terpenoids (mono-, di-, and tri-) have been observed to have antiurease activity [30], however, betuline and betulinic acid as pentacyclic triterpenes possess anti-HIV-1, antitumoural, anti-inflammatory and in vitro antimalarial effects [31]. Therefore, the activities of these compounds either singly or in concerted manner could be responsible for the observed biological effects.

\section{Conclusion}

In this study it was observed that the stem bark extract of Spondium mombin Linn is rich in the various compounds identified using GS-MS. The stem bark extract of this plant was found to have potential phytotoxic effect which can be further studied as an effective agent against parasitic plants. Though at high dose it could exert some lethal effect, but its medicinal potential can be cautiously harnessed for therapeutic gains.

\section{Acknowledgements}

We thank Natural Products Research and Disease Control Laboratory, University of Benin, Benin City, Nigeria, for the plant material and technical assistance during the preliminary stage of this study. Also, we appreciate the International Centre for Chemical and Biological Sciences (ICCBS) for the laboratory space, equipment and technical assistance.
Authors' contributions

KOO conceptualized, designed, carried out the study and prepared the manuscript. POU and MIC conceptualized, designed and supervised the study. The authors read and approved the final manuscript.

\section{Funding}

This work was supported by The World Academy of Science, TWAS-ICCBS Postgraduate Fellowship (FR Number: 3240287191) and Tertiary Education Trust Fund Research Grant awarded to Kissinger Obaogie Orumwensodia. The authors are immensely grateful.

Availability of data and materials

All data relating to this study have been included in this article.

\section{Declarations}

Ethics approval and consent to participate

Not applicable.

\section{Consent for publication}

Not applicable.

\section{Competing interests}

The authors declare no competing interest.

Received: 16 January 2021 Accepted: 23 June 2021

Published online: 30 June 2021

\section{References}

1. Njoku PC, Akumefula MI. Phytochemical and nutrient evaluation of Spondais mombin leaves. Pak J Nutr. 2007;6(6):613-5. https://doi.org/10.3923/pjn.2007. 613.615.

2. Aigbokhan El. Annotated checklist of vascular plants of southern Nigeria, a quick reference guide to the vascular plants of southern Nigeria: a systematic approach. Benin: Uniben Press; 2014.

3. Caraballo A, Caraballo B, Rodriquez-Acosta A. Preliminary assessment of medicinal plants used as antimalarials in the south-eastern Venezuelan Amazon. Rev Soc Bras Med Trop. 2004;37(2):186-8. https://doi.org/10.1590/ S0037-86822004000200016

4. Corthout J, Pieters LA, Claeys M, Vanden Berghe DA, Viletinck AJ. Antiviral Caffeoyl; Esters from Spondias mombin. Phytochemistry. 1992;31:79.

5. Corthout J, Pieters LA, Claeys M, Vanden Berghe DA, Viletinck AJ. Antibacterial and molluscicidal phenolic acid from Spondias mombin. Planta Med. 1994;60(05):460-3. https://doi.org/10.1055/s-2006-959532.

6. Villegas LF, Fernadz TD, Maldonado H, Torres R, Zavaleta A, Vaisberg AJ, et al. Evaluation of wounds healing of selected plants from Peru. Peru J Ethnopharmacol. 1997;55(3):193-200. https://doi.org/10.1016/S0378-8741 (96)01500-0.

7. Coates NJ, Gilpin ML, Gwynn MN, Lewis DE, Milner PH, Spear SR, et al. SB202742 a novel beta-lactamase inhibitor isolated from Spondias mombin. $J$ Nat Prod. 1994;57(5):654-7. https://doi.org/10.1021/np50107a016.

8. Orwa C, Mutua A, Kindt R, Jamnadass R, Anthony S. Spondias mombin Linn., a tree reference and selection guide version 4.0. Agroforestree Database. 2009. http://www.worldagroforestry.org/sites/treedbs/treedatabases.asp.

9. WHO. WHO traditional medicine strategy: 2014-2023: World Health Organization; 2013. https://apps.who.int/iris/handle/10665/92455. Accessed 14 Jan 2021

10. Karadeniz B, Ulker Z, Alpsoy L. Genotoxic and cytotoxic effects of storax in vitro. Toxicol Ind Health. 2013;29(2):181-6. https://doi.org/10.1177/074 8233711428642

11. MCLaughlin JL, Chang CJ, Smith DL. Bench Top Bioassays for the Discovery of Bioactive Natural Products. In: Atta-Ur-Rahaman, editor. Studies in Natural Products Chemistry. Amsterdam: Elsevier; 1991. p. 383-409.

12. Carballo JL, Hernández-Inda ZL, Pérez P, García-Grávalos MD. A comparison between two brine shrimp assays to detect in vitro cytotoxicity in marine natural products. BMC Biotechnol. 2002;2(1):17. https://doi.org/10.1186/14726750-2-17.

13. Osemwenkhae PO, Orumwensodia KO. In Vitro antioxidant effect and phytochemical composition of aqueous extract of Dennettia Tripetala seed. Nig J Life Sci. 2015;5:195-202. 
14. Rahman S, Parvez AK, Islam R, Khan MH. Antibacterial activity of natural spices on multiple drug resistant Escherichia coli isolated from drinking water. Bangladesh Ann Clin Microbiol Antimicrob. 2011;10(1):10. https://doi. org/10.1186/1476-0711-10-10

15. Hafidh RR, Abdulamir AS, Vern LS, Bakar FA, Abas F, Jahanshiri F, et al. Inhibition of growth of highly resistant bacterial and fungal pathogens by a natural product. Open Microbiol J. 2011;5(1):96-106. https://doi.org/10.21 74/1874285801105010096.

16. Oriakhi K, Orumwensodia KO. Combinatorial effect of Gallic acid and Catechin on some biochemical and pro-inflammatory markers in $\mathrm{CCl} 4$ -mediated hepatic damage in rats. Phytomedicine Plus. 2021;1(1):100017. https://doi.org/10.1016/j.phyplu.2020.100017.

17. Azizuddin, Choudhary Ml. Antibacterial, phytotoxic, insecticidal and cytotoxic potential of Vitex agnus-castus. J Med Plant Res. 2011;5:5642-5.

18. Asuquo OR, Ekanem TB, Eluwa MA, Oko OO, Ikpi DE. Evaluation of Toxicological Effects of Spondias mombin in Adult Male Wistar Rats. J Nat Sci Res. 2012;2:144-51.

19. Nwaogwugwu J, Uhegbu F, Okereke S, Egege A, Atasi O. Toxicological evaluation of aqueous leaf extract of Spondias mombin using albino rat. J Med Herbs Ethnomed. 2018:4:23-30.

20. Orhan I, Sener B. Lead compounds and drug candidates from some Turkish plants for human health: advances in Phytomedicine. 2nd ed. Oxford: Elsevier; 2006

21. Park SH, Sim YB, Lee JK, Kim SM, Kang YJ, Jung JS, et al. The analgesic effects and mechanisms of orally administered eugenol. Arch Pharm Res. 2011:34(3):501-7. https://doi.org/10.1007/s12272-011-0320-z.

22. Dal Bo W, Luiz AP, Martins DF, Mazzardo-Martins L, Santos AR. Eugeno reduces acute pain in mice by modulating the glutamatergic and tumor necrosis factor alpha (TNF-alpha) pathways. Fundam Clin Pharmacol. 2013, 27(5):517-25. https://doi.org/10.1111/j.1472-8206.2012.01052.x.

23. Fonseca DV, Salgado PR, Aragao Neto Hde C, Golzio AM, Caldas Filho MR, Melo CG, et al. Ortho-eugenol exhibits anti-nociceptive and antiinflammatory activities. Int Immunopharmacol. 2016;38:402-8. https://doi. org/10.1016/j.intimp.2016.06.005.

24. Pisano M, Pagnan G, Loi M, Mura ME, Tilocca MG, Palmieri G, et al. Antiproliferative and pro-apoptotic activity of eugenol-related biphenyls on malignant melanoma cells. Mol Cancer. 2007;6(1):8. https://doi.org/10.11 86/1476-4598-6-8.

25. Marchese A, Barbieri R, Coppo E, Orhan IE, Daglia M, Nabavi SF, et al. Antimicrobial activity of eugenol and essential oils containing eugenol: a mechanistic viewpoint. Crit Rev Microbiol. 2017;43(6):668-89. https://doi. org/10.1080/1040841X.2017.1295225.

26. Ahuja N, Batish DR, Singh HP, Kohli RK. Herbicidal activity of eugenol towards some grassy and broad-leaved weeds. J Pest Sci. 2015:88:209-18.

27. Fontana A, Spolaore B, de Laureto PP. The biological activities of protein/ oleic acid complexes reside in the fatty acid. Biochim Biophys Acta, Proteins Proteomics. 1834;2013:1125-43

28. Senyilmaz-Tiebe D, Pfaff DH, Virtue S, Schwarz KV, Fleming T, ALtamura S, et al. Dietary stearic acid regulates mitochondria in vivo in humans. Nat Commun. 2018:9(1):3129. https://doi.org/10.1038/s41467-018-05614-6.

29. Suttiarporn P, Chumpolsri W, Mahatheeranont S, Luangkamin S, Teepsawang S, Leardkamolkarn V. Structures of Phytosterols and triterpenoids with potential anti-Cancer activity in bran of black nonglutinous Rice. Nutrients. 2015;7(3):1672-87. https://doi.org/10.3390/nu7031 672.

30. Khan SS, Khan A, Khan A, Wadood A, Farooq U, Ahmed A, et al. Urease inhibitory activity of ursane type sulfated saponins from the aerial parts of Zygophyllum fabago Linn. Phytomedicine. 2014;21(3):379-82. https://doi. org/10.1016/j.phymed.2013.09.009.

31. Şoica CM, Dehelean CA, Peev C, Aluas M, Zupkó I, Kása P Jr, et al. Physicochemical comparison of betulinic acid, betulin and birch bark extract and in vitro investigation of their cytotoxic effects towards skin epidermoid carcinoma (A431), breast carcinoma (MCF7) and cervix adenocarcinoma (HeLa) cell lines. Nat Prod Res. 2012;26(10):968-74. https://doi.org/10.1 080/14786419.2010.545352

\section{Publisher's Note}

Springer Nature remains neutral with regard to jurisdictional claims in published maps and institutional affiliations.

\section{Submit your manuscript to a SpringerOpen ${ }^{\odot}$ journal and benefit from:}

- Convenient online submission

- Rigorous peer review

- Open access: articles freely available online

- High visibility within the field

- Retaining the copyright to your article

Submit your next manuscript at $\boldsymbol{\nabla}$ springeropen.com 